\title{
Cooperation stimulation mechanisms for wireless multihop networks: a survey
}

\begin{abstract}
In wireless multihop networks such as wireless sensor networks (WSNs) and mobile ad hoc networks (MANETs), nodes have to rely on their peer neighbours in transmitting packets to intended destinations. A successful rate of communication in these networks is assured if all nodes in the network fully cooperate to relay packets for each other. However, due to the existence of nodes with various motives, cooperativeness cannot be ensured and the communication goal is not achieved. Consequently, many cooperation stimulation approaches have been proposed to address node selfishness by using, broadly, incentive-based and punishment-based approaches. These schemes consist of several components including monitoring mechanisms, that need to be optimized in order to provide effective ways to detect and manage selfish nodes in the networks. This paper summarizes existing cooperation stimulation mechanisms and discusses important issues in this field such as false judgment and node collusion, whereby the root of these kinds of problems originates from the inability to obtain accurate evaluation on the behaviour of a node.
\end{abstract}

Keyword: Cooperation; Selfish behaviour; Wireless multihop networks; Incentive schemes; Punishment schemes; Game theory 